\title{
Is robotic mitral valve repair a reproducible approach?
}

\author{
Wen Cheng, MD, Gregory P. Fontana, MD, Michele A. De Robertis, RN, James Mirocha, MS, \\ Lawrence S. C. Czer, MD, FACC, Robert M. Kass, MD, and Alfredo Trento, MD, FACS
} Objective: We sought to review the surgical outcomes of our initial 120 robotic mitral valve repairs from June
2005 through April 2009.

\begin{abstract}
Methods: The initial 74 repairs were performed with the first-generation da Vinci robot (Intuitive Surgical, Inc, Sunny Vale, Calif), and the last 46 were performed with the da Vinci Si HD model. All patients received an annuloplasty band and 1 or more of the following: leaflet resection; annuloplasty; basal chord transposition, polytetrafluoroethylene neochordal replacement, or both; and edge-to-edge repair.
\end{abstract}

Results: The overall mean age was $58.4 \pm 10.5$ years, and $64 \%$ were male. There was $1(0.8 \%)$ hospital mortality. Five patients required mitral valve replacement for a failed repair. Another patient had mitral valve rerepair on postoperative day 2. Except for 2 early reoperations for postoperative bleeding, all of the complications and failed repairs requiring operative revision occurred with the original robot. Postdischarge transthoracic echocardiographic follow-up was available on 107 (93\%) of 115 patients, with a median follow-up of 321 days. None to mild mitral regurgitation was seen in $102(89 \%)$ patients, moderate mitral regurgitation was seen in $9(8.4 \%)$ patients, and severe mitral regurgitation was seen in $3(2.8 \%)$, with 1 patient undergoing mitral valve replacement and 2 patients being medically managed.

Conclusions: The majority of complications and all the repeat operations for failed mitral valve repair occurred with the older-model da Vinci robot. The newer da Vinci Si HD system, with the addition of an adjustable left atrial roof retractor, improves mitral valve exposure, enhancing the surgeon's ability to repair and test the valve. We have progressed to successful repair of all types of degenerative mitral valve pathology and have found the approach reproducible. (J Thorac Cardiovasc Surg 2010;139:628-33)

Since Dr Carpentier's introduction of the "French correction," mitral valve repair for degenerative (myxomatous) disease has become the gold standard approach. ${ }^{1}$ In the mid-1990s, several surgical groups developed and refined techniques with small thoracotomy endoscopic approaches, achieving results equal to those seen with the traditional sternotomy operation. ${ }^{2-4}$ Dr Chitwood further refined the endoscopic approach, taking advantage of the da Vinci Robotic Surgical System (Intuitive Surgical, Inc, Sunny Vale, Calif) and its advanced robotic technology to repair complex mitral valve pathology ${ }^{5-7}$ The excellent clinical outcome was built on the foundation of stepwise improvement in technique with a gradual learning curve. ${ }^{7,8}$

In 2005, we embarked on the process of developing a robotic mitral valve surgery program. We had a small experi-

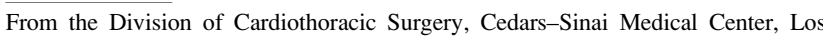
Angeles, Calif.

Disclosures: Lawrence Czer reports equity and grant support from St Jude.

Supported by the Sanders Family Trust.

Read at the Thirty-fifth Annual Meeting of The Western Thoracic Surgical Association, Banff, Alberta, Canada, June 24-27, 2009.

Received for publication June 23, 2009; revisions received Oct 14, 2009; accepted for publication Oct 25, 2009; available ahead of print Dec 28, 2009.

Address for reprints: Alfredo Trento, MD, Division of Cardiothoracic Surgery, Cedars-Sinai Medical Center, 8700 Beverly Blvd, N.T. Room 6215, Los Angeles, CA 90048 (E-mail: alfredo.trento@cshs.org).

$0022-5223 / \$ 36.00$

Copyright (c) 2010 by The American Association for Thoracic Surgery doi:10.1016/j.jtcvs.2009.10.047
}

ence with a right minithoracotomy for mitral surgery. Our team's orientation and training included programmatic and simulation training at the surgical Robotic Training Center at East Carolina University and visits to other high-volume centers to observe robotic mitral surgery cases. On-site proctoring by Intuitive Surgical, Inc, for our initial cases supplemented the training we received. This report describes the clinical outcomes in our first 120 patients undergoing robot-assisted mitral valve repair.

\section{MATERIALS AND METHODS}

From June 2005 through April 2009, 120 patients underwent intent-totreat endoscopic robotic mitral valve repair. There was 1 intraoperative conversion to a right minithoracotomy before completion of the mitral repair. This was due to external instrument conflicts of the robotic arms that could not be resolved because of the space limitations of working in a small right hemithorax. Otherwise, 119 patients achieved successful completion of the robotic mitral valve repair. Robotic mitral valve repair represents an increasing proportion of a single surgeon's (AT) experience with various approaches, minithoracotomy, and sternotomy during this period (Figure 1).

Patients with heavily calcified mitral valve annuli, dense right pleural adhesions, and severe aortoiliac atherosclerosis were approached through a median sternotomy. Patients with prior median sternotomy were approached through a minithoracotomy or redo median sternotomy. Patients likely to require mitral valve replacement, such as those with rheumatic mitral disease, endocarditis, and restrictive posterior leaflet pathology, tended to be approached through a minithoracotomy or median sternotomy.

The data presented in this retrospective review were obtained from an institutionally supported Cardiothoracic Surgery Quality Assurance Database. The study was approved by the Cedars-Sinai Institutional Review Board 


\section{Abbreviations and Acronyms \\ $\mathrm{MR}=$ mitral regurgitation \\ $\mathrm{SAM}=$ systolic anterior motion \\ TEE $=$ transesophageal echocardiography}

and granted a waiver of informed consent given the minimal risk imposed on the patients and given that it would not adversely affect the rights and welfare of these patients who were deidentified for this group analysis.

\section{Operative Technique}

Our current standard practice is to intubate the patient with a double-lumen endotracheal tube and place a coronary sinus cardioplegia catheter during transesophageal echocardiographic (TEE) guidance. In our early experience, contrast dye was injected to check the catheter position during fluoroscopic analysis. In addition, a pulmonary artery drain was placed for left heart drainage. Both of these techniques were later believed to be unnecessary and discontinued. With TEE guidance, more than $90 \%$ of patients have had successful placement of a coronary sinus catheter for retrograde cardioplegia delivery. There were no instances of coronary sinus damage or rupture from either catheter placement or use.

After the patient is positioned supine with the right side of the chest elevated, the right lung is deflated, and a 3- to 4-cm right inframammary incision through the fourth or fifth intercostal space is made as the working port. Trocars for the robotic instrument arms, 14-gauge angiocatheters for traction sutures, and a 20F DLP (Medtronic, Inc, Minneapolis, Minn) intracardiac sump drain are inserted through a separate stab-wound incision. Our first 74 cases were performed with the original version of the da Vinci Robotic Surgical System. For these first cases, the handle of the atrial septal roof retractor (Cardiovations, Irvine, Calif) was inserted just lateral to the right intrathoracic artery. The last 46 cases were performed with the second-generation da Vinci Si HD, with a fourth articulating arm as a movable left atrial roof retractor.

The patient is heparinized and the femoral vessels are cannulated for cardiopulmonary bypass. A 2 -stage venous cannula is placed through the femoral vein under TEE guidance to ensure proper placement for venous drainage with vacuum assistance.

The pericardium is incised ventral to the phrenic nerve. A transthoracic aortic clamp (Scanlan International, Minneapolis, Minn) is positioned through the third intercostal space in the midaxillary line. An ascending aortic root vent (DLP 14-gauage 7F; Medtronic, Inc) is placed under direct vision. The da Vinci robot is positioned to the patient's left side, and the instrument arms are placed endoscopically. Bypass is established, and the patient is cooled to $32^{\circ} \mathrm{C}$. Once cold, the crossclamp is positioned and clamped under endoscopic vision. One hundred twenty milliliters of cold blood cardioplegia ( $\mathrm{K}^{+}$, mannitol, $\mathrm{HCO}_{3}$ mixture) is infused directly through the aortic root. Then retrograde crystalloid cardioplegia is gravity dripped into the coronary sinus catheter limited to $1 \mathrm{~L}$ per patient. If the coronary sinus catheter could not be placed or becomes dislodged, multiple 200-mL doses of cold antegrade crystalloid cardioplegia would be administered by the anesthesiologist about every 20 minutes after crossclamping to supplement the initial dose of cold blood cardioplegia. Once asystole is achieved, a standard left interatrial incision is made with the robot, and the left atrial roof retractor is positioned for mitral valve exposure.

If a concomitant CryoMaze procedure is performed, the Cryocath (Medtronic CryoCath LP, Montreal, Quebec, Canada) is used to create a pulmonary vein isolation box lesion and a connecting lesion to the mitral annulus. Then standard mitral repair techniques are used for leaflet resections and reconstructions with suture knot tying done intracorporeally with the robotic arms. If polytetrafluoroethylene neochordae (Gore-Tex suture; W. L. Gore and Associates, Inc, Flagstaff, Ariz) are placed, knot tying is done extracor-

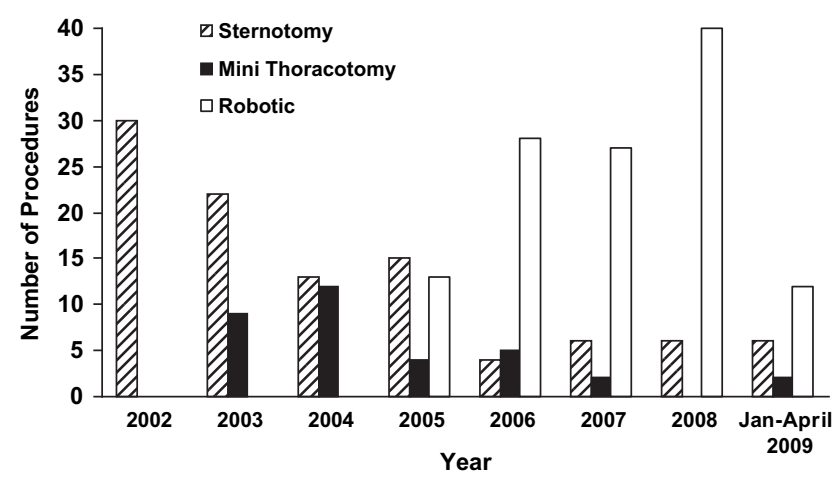

FIGURE 1. The bar graph shows the trends in the surgical approach used for mitral valve repair surgery at our institution from 2002 to the present.

poreally by the bedside assistant surgeon using a shafted knot pusher. All of the annuloplasty bands used were partial rings. Initially, we used the Tailor band (St Jude Medical, Inc, St Paul, Minn) but later switched to the ATS band (ATS Medical, Inc, Minneapolis, Minn) because it crimped less when secured with either of 2 techniques: nitinol U-clips (Medtronic, Inc) or 2-0 Cardioflon sutures (Peters, Inc, Paris, France). Left atriotomy closure is done with a running $4-0$ polytetrafluoroethylene sutures. Deairing the heart is performed with active suction on the aortic root vent with increasing controlled ejection while at the same time actively ventilating and decreasing pump flow. The completeness of deairing is monitored by using TEE. Humidified $\mathrm{CO}_{2}$ is also insufflated into the operative field through the camera port.

\section{Follow-up}

All surviving patients $(\mathrm{n}=119)$ were examined and clinically evaluated within 2 weeks after hospital discharge. Further clinical follow-up was obtained through direct patient contact or through the patients' referring physicians. Echocardiographic follow-up reports are routinely obtained from the referring physicians. Median echocardiographic follow-up was 321 days (mean \pm standard deviation, $372.9 \pm 331.6$ days).

\section{Statistical Methods}

Numeric variables are summarized as means \pm standard deviations or medians (ranges). Categorical variables are summarized as frequencies (percentages).

\section{Patient Characteristics}

Table 1 lists the preoperative patient characteristics. All patients underwent functional valve assessment by means of intraoperative TEE, and the valve pathology is confirmed at the time of the operation (Table 2).

\section{RESULTS}

Table 3 lists the operative details of all 120 patients. All patients received an annuloplasty band. Five patients received only a simple band. One third (40 [33.3\%]) of the patients had a leaflet resection and annuloplasty. There were 40 patients who had chordal procedures, either the transfer of basal chords $(n=12)$ or implantation of polytetrafluoroethylene chords $(\mathrm{n}=28)$.

Two patients were converted to mitral valve replacement at the time of the initial operation for failed repairs. Patient 33 had posterior leaflet restriction after repair and proceeded to porcine valve replacement through a median sternotomy. 
TABLE 1. Preoperative demographics

\begin{tabular}{lc}
\hline Characteristics & \\
Age $(\mathrm{y})$, mean $\pm \mathrm{SD}$ & $58.4 \pm 10.5$ \\
Age $(\mathrm{y})$, range & $33-86$ \\
Sex & \\
$\quad$ Male & $77(64.2)$ \\
$\quad$ Female & $43(35.8)$ \\
Body mass index $\left(\mathrm{kg} / \mathrm{km}^{2}\right)$, mean $\pm \mathrm{SD}$ & $25.4 \pm 4.0$ \\
Body mass index, range & $18.8-37.3$ \\
New York Heart Association class & \\
I & $26(21.7)$ \\
$\quad$ II & $43(35.8)$ \\
$\quad$ III & $45(37.5)$ \\
IV & $6(5.0)$ \\
Comorbidities & \\
Congestive heart failure & $94(78.3)$ \\
Diabetes & $3(2.5)$ \\
Hypertension & $37(30.8)$ \\
Serum creatinine $>2.0$ mg/dL & $0(0)$ \\
Atrial fibrillation/flutter & $29(24.2)$ \\
Stroke & $0(0)$ \\
Transient ischemic attack & $4(3.3)$ \\
Peripheral vascular disease & $2(1.7)$ \\
Smoking history & $30(25.0)$ \\
Previous cardiac surgery & $1(0.8)$ \\
Echocardiographic findings & \\
LV ejection fraction, mean $\pm \mathrm{SD}$ & $61.2 \% \pm 7.7 \%$ \\
LV ejection fraction, range & $38 \%-86 \%$ \\
Severe mitral regurgitation & $120(100)$ \\
\hline
\end{tabular}

Values are presented as numbers (percentages), unless indicated otherwise. $S D$, Standard deviation; $L V$, left ventricular.

Patient 68 had a failed repair of a Barlow valve caused by postrepair left ventricular outflow obstruction associated with systolic anterior motion (SAM) of the mitral valve and had placement of a porcine valve through the minithoracotomy incision.

One quarter (29 [24.2\%]) of the patients had a concomitant left-sided CryoMaze procedure, with 15 (12.5\%) having left atrial appendage exclusion with a running 4-0 polytetrafluoroethylene suture. Eight $(6.7 \%)$ patients had

TABLE 2. Echocardiographic and operative mitral valve characteristics $(\mathbf{n}=\mathbf{1 2 0})$

\begin{tabular}{lc}
\hline Carpentier classification & \\
Type 1 & $5(4.2)$ \\
Type 2 & $115(95.8)$ \\
Anterior prolapse & $7(5.8)$ \\
Posterior prolapse & $86(71.7)$ \\
Bileaflet prolapse & $22(18.3)$ \\
Type 3 & $0(0)$ \\
Valve pathology & \\
Degenerative & \\
Fibroelastic deficiency & $106(88.3)$ \\
Barlow & $6(5.0)$ \\
Endocarditis & $8(6.7)$ \\
\hline
\end{tabular}

Values are presented as numbers (percentages).
TABLE 3. Operative characteristics

\begin{tabular}{lc}
\hline Mitral valve repair type & \\
Annuloplasty band & $120(100)$ \\
Band size (mm), median (range) & $33(23-39)$ \\
Annuloplasty band alone & $5(4.2)$ \\
Edge-to-edge & $7(5.8)$ \\
Edge-to-edge + chordal procedure & $4(3.3)$ \\
Leaflet resection & $28(23.3)$ \\
Leaflet resection + annuloplasty & $40(33.3)$ \\
Leaflet resection + chordal procedure \pm annuloplasty & $16(13.3)$ \\
Chordal procedure (no leaflet resection) & $20(16.7)$ \\
Polytetrafluoroethylene neochordae & $28(23.3)$ \\
Concomitant procedures & \\
CryoMaze atrial fibrillation ablation & $29(24.2)$ \\
Exclusion of left atrial appendage & $15(12.5)$ \\
Atrial septal defect closure & $1(0.8)$ \\
Patent foramen ovale closure & $8(6.7)$ \\
Perfusion details & \\
Cardiopulmonary bypass time (min), mean \pm SD & $156.9 \pm 43.5$ \\
Crossclamp time (min), mean \pm SD & $116.6 \pm 30.6$ \\
\hline Values are presented as numbers (percentages), unless indicated otherwise. SD, Stan- \\
dard deviation.
\end{tabular}

patent foramen ovale closure, and 1 had an atrial septal defect closure. The mean crossclamp time was $116.6 \pm 30.6$ minutes. There were 41 patients with crossclamp times of greater than 120 minutes, and 5 patients with crossclamp times of greater than 180 minutes. The longest crossclamp time was 221 minutes, and this patient represented the only surgical death in the series. Patients with prolonged crossclamp times had more complex procedures, often with an associated CryoMaze procedure. There were 2 repair failures requiring subsequent valve replacement in these 41 patients. Of the remaining 38 patients, all are asymptomatic and in New York Heart Association class I. We are currently following 1 patient with recurrent severe mitral regurgitation (MR) and 1 patient with moderate MR.

\section{Mortality and Morbidity}

Postoperative complications are listed in Table 4. There was $1(0.8 \%)$ early death, and this occurred in patient 4 , who had severe myocardial dysfunction after repair. Despite ECMO followed by conversion to biventricular assist device support, the patient died on postoperative day 3 from multisystem organ dysfunction. The final pathology report confirmed a massive left ventricular hemorrhagic infarction. In our first 4 patients, we did not place a coronary sinus catheter for retrograde cardioplegia delivery. Our myocardial protection consisted of $120 \mathrm{~mL}$ of antegrade cardioplegia followed by $1 \mathrm{~L}$ of cold crystalloid cardioplegia. The patient had bileaflet prolapse requiring a posterior leaflet resection and transposition of 4 sets of basal chords to the anterior leaflet. This took 221 minutes of crossclamp time. Despite no obvious electrical activity on the monitor during the period of crossclamping, it is very likely that the myocardial protection was not sufficient. 
TABLE 4. Postoperative outcomes and complications

\begin{tabular}{lc}
\hline 30-d Mortality & $1(0.8)$ \\
Late mortality & $0(0)$ \\
Myocardial infarction & $1(0.8)$ \\
Stroke & $2(1.7)$ \\
Reversible neurologic injury & $2(1.7)$ \\
Prolonged mechanical ventilation $(>48 \mathrm{~h})$ & $2(1.7)$ \\
Bleeding requiring reoperation & $6(5.0)$ \\
Any blood product transfusion & $45(37.5)$ \\
$\quad$ pRBC & $23(19.1)$ \\
FFP & $32(26.7)$ \\
$\quad$ Platelets & $36(30.0)$ \\
Infection & $0(0)$ \\
Serum creatinine $>2.0$ mg/dL & $3(2.5)$ \\
Atrial fibrillation/flutter & $12(10.0)$ \\
Pleural effusion & $2(1.7)$ \\
Diaphragm paralysis & $1(0.8)$ \\
Needle lost in left inferior pulmonary vein & $1(0.8)$ \\
Hospital length of stay $(d)$, mean \pm SD & $6.3 \pm 3.9$ \\
Readmission within $30 \mathrm{~d}$ & $1(0.8)$ \\
Repair failures and second operation & \\
$\quad$ Mitral replacement through sternotomy & $4(3.3)$ \\
Mitral valve replacement through minithoracotomy & $1(0.8)$ \\
Mitral valve rerepair through minithoracotomy & $1(0.8)$ \\
\hline Values are presented as numbers (percentages), unless indicated otherwise. $p R B C$, \\
Packed red blood cells; $F F P$, fresh frozen plasma; $S D$, standard deviation \\
\end{tabular}

After this patient, we changed our myocardial protection strategy to include coronary sinus catheter placement for delivery of cold retrograde crystalloid cardioplegia, up to $1 \mathrm{~L}$ total, administered by means of continuous drip. We currently use multiple doses of cold antegrade crystalloid cardioplegia every 20 minutes when retrograde access for cardioplegia delivery is not available. Of the first 3 patients who received only $120 \mathrm{~mL}$ of antegrade blood cardioplegia followed by $1 \mathrm{~L}$ of crystalloid cardioplegia as an initial dose, all are doing well at 4 years' follow-up, with ejection fractions of $55 \%$ to $62 \%$ and no more than trace MR at the latest follow-up. More than $90 \%$ of patients subsequently had retrograde cardioplegia or multiple doses of antegrade crystalloid cardioplegia, with no problems encountered in myocardial protection since the change.

There was no late mortality in this series. Patient 39 had a small, less than $1-\mathrm{cm}$ laceration to the dome of the diaphragm through the roof of the liver. This led to abdominal bleeding (heparinized), which was noted after the mitral repair was completed. The mitral repair was intact, but he experienced cardiac arrest after protamine had been administered and required a laparotomy to repair the liver laceration. $\mathrm{He}$ has a permanent right hemisphere stroke with moderate residual disability. It is likely that the diaphragm laceration and liver injury occurred with placement of the robotic arms.

Overall, there were 2 patients with strokes who have persistent neurologic deficits, and $2(1.7 \%)$ patients experienced a transient neurologic event with full resolution. Six $(5.0 \%)$ patients returned to the operating room for postsur- gical bleeding or for evacuation of retained hemothorax during their initial hospital stay. Three patients had postoperative creatinine values of greater than 2.0 during their hospitalization, with 1 patient requiring temporary hemodialysis. There were no femoral artery or vein complications and no lymphoceles requiring surgical intervention. The hospital length of stay was $6.3 \pm 3.9$ days, with only $1(0.8 \%)$ patient requiring readmission within 30 days.

In addition to the 2 patients converted to mitral valve replacement at the time of the initial failed robotic mitral valve repairs, there have been 3 subsequent operations for early ( $<30$ day) repair failure, with $2(1.7 \%)$ mitral valve replacements and $1(0.8 \%)$ repeat repair through a minithoracotomy. All of these repair failures occurred in the first 74 patients who underwent repair with the older-model da Vinci robot. Since the introduction of the da Vinci Si HD, there have been no major complications, with only $2(1.7 \%)$ patients returning to the operating room for postoperative bleeding.

\section{Echocardiographic Studies and Patient Follow-up}

All 120 patients had severe MR, as determined by means of preoperative transthoracic echocardiographic analysis (Table 5) that was confirmed with prepump TEE. There were 2 patients with moderately severe MR, as determined by postpump TEE, who then had the valve replaced. Four additional patients had moderate-to-severe MR during the hospitalization.

Patient 25 underwent rerepair of the mitral valve through the original minithoracotomy incision. At the time of the operation, the band and part of the posterior leaflet were partially dehisced from the mitral annulus. This band dehiscence was due to placement of the nitinol clips insufficiently deep in the mitral annulus.

Patient 53 had a restricted posterior leaflet as the source of his recurrent MR and had a mitral valve replacement through a median sternotomy on postoperative day 3 .

Patient 58 had worsening SAM that was unresponsive to medical management and had mitral valve replacement on postoperative day 6 through a minithoracotomy. In this patient we treated severe prolapse of the posterior leaflet (P2 and P3) with multiple polytetrafluoroethylene chords and a 35-mm ATS band. His initial postoperative TEE showed only trace MR, but he later developed SAM. In retrospect, the surgeon believed he should have resected some of the posterior leaflet to reduce its height, thereby minimizing the chance for SAM.

Patient 5 had severe MR on predischarge transthoracic echocardiographic analysis, which remains unchanged. $\mathrm{He}$ is currently being medically managed and is asymptomatic at 3 years' follow-up.

Patient 47 had mitral valve replacement on day 41 performed through a median sternotomy. At the time of the first operation, he was found to have a Barlow-type valve with bileaflet prolapse. Two polytetrafluoroethylene chords 
TABLE 5. Echocardiographic data

\begin{tabular}{|c|c|c|c|c|}
\hline & Preoperative TTE $(n=120)$ & Postpump TEE $(\mathrm{n}=119)$ & In-hospital TTE $(\mathrm{n}=20)$ & Postdischarge TTE $(n=107)$ \\
\hline \multicolumn{5}{|l|}{ MR severity } \\
\hline None & & $65(54.2)$ & $5(25)$ & $35(32.7)$ \\
\hline Trace & & $33(27.5)$ & $7(35)$ & $32(29.9)$ \\
\hline Mild & & $19(15.8)$ & $3(15)$ & $28(26.2)$ \\
\hline Moderate & & $2(1.7)$ & $2(10)$ & $9(8.4)$ \\
\hline Severe & $120(100)$ & $0(0)$ & $3(15)$ & $3(2.8)$ \\
\hline da Vinci & Preoperative TTE $(n=74)$ & Postpump TEE $(n=73)$ & In-hospital TTE $(\mathrm{n}=16)$ & Out-of-hospital TTE $(n=66)$ \\
\hline \multicolumn{5}{|l|}{ MR severity } \\
\hline None & & 36 (49.3) & $4(25)$ & $20(30.3)$ \\
\hline Trace & & $23(31.5)$ & $4(25)$ & $18(27.3)$ \\
\hline Mild & & $12(16.4)$ & $4(25)$ & $20(30.3)$ \\
\hline Moderate & & $2(2.7)$ & $1(6.2)$ & $5(7.6)$ \\
\hline Severe & $74(100)$ & $0(0)$ & $3(18.8)$ & $3(4.5)$ \\
\hline da Vinci Si HD & Preoperative TTE $(n=46)$ & Postpump TEE $(n=46)$ & In-hospital TTE $(\mathbf{n}=5)$ & Out-of-hospital TTE $(n=41)$ \\
\hline \multicolumn{5}{|l|}{ MR severity } \\
\hline None & & $29(63.0)$ & $1(20)$ & 15 (36.6) \\
\hline Trace & & $10(21.7)$ & $3(60)$ & $14(34.1)$ \\
\hline Mild & & $7(15.2)$ & $0(0)$ & $8(19.5)$ \\
\hline Moderate & & $0(0)$ & $1(20)$ & $4(9.8)$ \\
\hline Severe & $46(100)$ & $0(0)$ & $0(0)$ & $0(0)$ \\
\hline
\end{tabular}

Values are presented as numbers (percentages). TTE, Transthoracic echocardiography; TEE, transesophageal echocardiography; $M R$, mitral regurgitation.

were placed to the anterior leaflet and 1 to the posterior leaflet, with a triangular resection of the middle scallop of the posterior leaflet (P2). A number 35 ATS band was placed. Intraoperative TEE confirmed trace residual MR. The postpump mitral valve annular diameter was $26 \mathrm{~mm}$. Myocardial protection was with our standard $120 \mathrm{~mL}$ of antegrade blood cardioplegia followed by a total of $850 \mathrm{~mL}$ of cold retrograde crystalloid cardioplegia administered by means of continuous drip. Crossclamp time was 2 hours and 14 minutes with a perfusion time of 3 hours and 2 minutes. The patient required no inotropic support after the operation. Discharge was delayed until postoperative day 9 because of postoperative atrial fibrillation, which required warfarin anticoagulation but no antiarrhythmic drug therapy. This patient later had unexpected cardiomyopathy after hospital discharge, with an ejection fraction of $25 \%$ at the time of his valve replacement. We do not believe myocardial protection was a contributing factor because ventricular function was normal after valve repair surgery and required no inotropic support. At the time of the operation, the preoperative TEE showed dehiscence of the band from the posterior annulus when compared with the postrepair TEE from 41 days previously. The prepump mitral annular diameter was now $37.5 \mathrm{~mm}$, more than $11 \mathrm{~mm}$ larger than the diameter after the first repair. This was confirmed by the surgeon at reoperation, with band dehiscence from the posterior annulus caused by annular dilatation. We are currently following 2 additional patients with recurrent severe MR medically managed and asymptomatic at last follow-up.
The 1 death and 7 failed repairs all occurred in the first 74 patients who underwent repair with the older-model da Vinci robot that lacked the adjustable left atrial roof retractor. The last 46 patients in this series, who underwent repair with the da Vinci Si HD, have had no mitral valve reinterventions to date. However, there are 4 patients in this later cohort who we are following with moderate MR. One of these patients had a P2 triangular resection and placement of an ATS band. One of his follow-up echocardiograms showed mildto-moderate MR. He is currently asymptomatic. The other 3 patients had annular dilatation and were treated with ATS bands. They currently have moderate MR and are asymptomatic with medical management. Our present practice is to use a complete ring for these patients because a partial ring might not provide the best annular stabilization at different loading conditions for these patients.

\section{DISCUSSION}

Robotic mitral valve repair has been performed with excellent results by a limited number of surgeons who have had an extensive background in minimally invasive mitral valve surgery. ${ }^{4-8}$ In October 2000, a US Food and Drug Administration-approved, multicenter, phase II trial (G000295) was initiated, with 10 centers evaluating the da Vinci surgical system. The results of this multicenter trial were reported to the surgical community at the American Association for Thoracic Surgery meeting in Boston in 2003. Subsequently, large clinical series with excellent clinical and echocardiographic outcomes have been reported. ${ }^{4-8}$ 
We initiated our robotic mitral surgery program in 2005. Our surgical team was initially trained at the Surgical Robotic Training Center at East Carolina University using a curriculum-based program that included case observation, didactic sessions, and both inanimate and cadaver models. Further training, sponsored by Intuitive Surgical, Inc, included robotic case observations in Atlanta, Georgia, and Spokane, Washington. Our initial cases were proctored by representatives of Intuitive Surgical, Inc.

Our team's learning curve can be separated into 2 phases. The initial phase was the first 74 patients with the oldermodel da Vinci robot that lacked the adjustable fourth articulating arm, which allows the surgeon to adjust left atrial exposure as he or she works on different areas of the mitral valve. The second phase was the last 46 patients with the newer-model da Vinci Si HD with an adjustable left atrial retractor and improved 3-dimensional visualization. All of the failed repairs requiring 5 valve replacements and 1 rerepair occurred with the earlier-generation robot. Two additional patients are being medically managed with severe MR. Of the 46 patients undergoing repair with the da Vinci Si HD, none have severe MR at the last follow-up, with 4 (9.8\%) being followed for moderate MR.

The difficulty we experienced maintaining optimal exposure of the mitral valve for the entire operation led to technical difficulty with certain aspects of the valve repair, including band placement, leaflet repair, and testing of the valve. The da Vinci Si HD, with its adjustable atrial roof retractor, provides mitral valve exposure that can be adjusted as a surgeon works on different areas of the mitral valve. It can be removed completely or the retraction can be decreased when the surgeon tests the valve repair with saline injection. We believe that this flexibility allows more secure and accurate placement of the stitches used for the repair. Moreover, the surgeon has an enhanced ability to study the valve and determine a plan for valve repair and then accurately test the result. The previous da Vinci robot model for mitral valve exposure relied on a left atrial retractor blade connected to a metal rod placed percutaneously through the fifth intercostal space and held with an adjustable metal arm attached to the side rail of the operating room bed. Adjustments were impossible during the course of the operation unless the robot was repositioned away from the patient's left side. After the exposure was achieved, we would then need to reposition the robot and replace the instruments and camera. Rather than perform these time-consuming steps, the surgeon would often proceed with less than optimal visualization. We believe that the 2 instances of ring dehiscence were due to poorly placed annuloplasty stitches caused by poor exposure. Having complete freedom to adjust our valve exposure during our later cases allowed us to implement advanced anterior leaflet repair techniques, such as chordal transposition and placement of polytetrafluoroethylene neochords. Since the introduction of the da
Vinci Si HD, there have been no repair failures, and the echocardiographic follow-up has documented a low incidence of residual or recurrent MR.

The majority of our complications occurred in our first 74 patients. Our only death occurred in patient 4 , who underwent a complicated repair with a long crossclamp time. We relied on a single $120-\mathrm{mL}$ dose of antegrade blood cardioplegia followed by $1 \mathrm{~L}$ of antegrade crystalloid cardioplegia. On unclamping, there was severe left ventricular dysfunction, which did not improve and later required extracorporeal membrane oxygenation support.

Four strokes have occurred in this entire series, with 2 completely resolved and 2 partially resolved. We routinely evaluate the thoracic aorta down to the iliac arteries with a computed tomographic angiogram, looking for calcific vascular disease that would increase the risk of retrograde femoral perfusion in patients older than 70 years and in patients younger than 70 years who have risk factors for vascular disease, such as diabetes, hypertension, and a significant smoking history. Although not eliminating retrograde aortic perfusion as a source of cerebral emboli and neurologic deficits, we believe that this will minimize the risk. We insufflate $\mathrm{CO}_{2}$ continuously through the camera introducer, flooding the operative field with $\mathrm{CO}_{2}$ in an attempt to reduce left atrial and ventricular air bubbles. These neurologic deficits could still be due to inadequate deairing or retained particulate embolus from the operative field. We are very careful with broken sutures and other debris encountered during the repair, ensuring that we remove all these sources of potential embolus.

Overall, we are satisfied with our clinical outcomes after robotic mitral valve repair. We believe that robotic mitral valve repair since the introduction of the da Vinci Si HD, the latest-generation robotic surgical system, has become easier to perform and the quality of the valve repair has improved. Robotic mitral valve repair has become a reproducible approach. Longer-term follow-up will be needed to confirm the durability of our robotic mitral valve repairs.

\section{References}

1. Carpentier A. Cardiac valve surgery-the "French correction"' J Thorac Cardiovasc Surg. 1983;86:323-37.

2. Casselman FP, Van Slycke S, Dom H, Lambrechts DL, Vermenlen Y, Vanermen H. Endoscopic mitral valve repair: feasible, reproducible and durable. J Thorac Cardiovasc Surg. 2003;125:273-82.

3. Mehmanesh H, Henze R, Lange R. Totally endoscopic mitral valve repair. J Thorac Cardiovasc Surg. 2002;123:96-7.

4. Felger JE, Chitwood WR Jr, Nifong LW, Halbert D. Evolution of mitral valve surgery: towards a totally endoscopic approach. Ann Thorac Surg. 2001;72:1203-9.

5. Nifong LW, Chu VF, Bailey BM, Maziarz DM, Sorrel VL, Halbert D, et al. Robotic mitral valve repair: experience with the da Vinci system. Ann Thorac Surg. 2003; 75:438-43.

6. Nifong LW, Chitwood WR, Pappas PS, Smith CR, Argenziano M, Starnes VA et al. Robotic mitral valve surgery: a United States multicenter trial. J Thorac Cardiovasc Surg. 2005;129:1395-404

7. Chitwood WR, Rodriguez E, Chu MWA, Hassan A, Ferguson TB, Vos PW, et al Robotic mitral valve repairs in 300 patients: a single-center experience. J Thorac Cardiovasc Surg. 2008;136:436-41.

8. Murphy DA, Miller JS, Langford DA, Snyder AB. Endoscopic robotic mitral valve surgery. J Thorac Cardiovasc Surg. 2006;132:776-81. 